\title{
Identification of candidate genes linking systemic inflammation to atherosclerosis; results of a human in vivo LPS infusion study
}

Suthesh Sivapalaratnam ${ }^{1 *}{ }^{*}$, Rosienne Farrugia ${ }^{2 \dagger}$, Max Nieuwdorp ${ }^{1}$, Cordelia F Langford ${ }^{3}$, Rachel T van Beem ${ }^{4}$, Stephanie Maiwald ${ }^{1}$, Jaap Jan Zwaginga ${ }^{4}$, Arief Gusnanto ${ }^{5}$, Nicholas A Watkins ${ }^{2,6}$, Mieke D Trip ${ }^{1}$ and Willem H Ouwehand ${ }^{2,3,6}$

\begin{abstract}
Background: It is widely accepted that atherosclerosis and inflammation are intimately linked. Monocytes play a key role in both of these processes and we hypothesized that activation of inflammatory pathways in monocytes would lead to, among others, proatherogenic changes in the monocyte transcriptome. Such differentially expressed genes in circulating monocytes would be strong candidates for further investigation in disease association studies.

Methods: Endotoxin, lipopolysaccharide (LPS), or saline control was infused in healthy volunteers. Monocyte RNA was isolated, processed and hybridized to Hver 2.1.1 spotted cDNA microarrays. Differential expression of key genes was confirmed by RT-PCR and results were compared to in vitro data obtained by our group to identify candidate genes.

Results: All subjects who received LPS experienced the anticipated clinical response indicating successful stimulation. One hour after LPS infusion, 11 genes were identified as being differentially expressed; 1 down regulated and 10 up regulated. Four hours after LPS infusion, 28 genes were identified as being differentially expressed; 3 being down regulated and 25 up regulated. No genes were significantly differentially expressed following saline infusion. Comparison with results obtained in in vitro experiments lead to the identification of 6 strong candidate genes (BATF, BID, C3aR1, ILIRN, SEC61B and SLC43A3)
\end{abstract}

Conclusion: In vivo endotoxin exposure of healthy individuals resulted in the identification of several candidate genes through which systemic inflammation links to atherosclerosis.

Keywords: Human, Monocytes, LPS infusion, Transcriptome, In Vivo

\section{Background}

Inflammation and atherosclerosis are closely linked. In patients with chronic inflammation due to disorders such as rheumatoid arthritis or systemic lupus erythematodus (SLE), the incidence of cardiovascular disease (CVD) is 2 to 50 -fold higher [1,2]. Even after correction for traditional risk factors, patients with chronic inflammatory disorders have accelerated plaque progression

\footnotetext{
* Correspondence: s.sivapalaratnam@amc.uva.nl

+ Contributed equally

'Department of Vascular Medicine, Academic Medical Center, Meibergdreef

15, 1105 AZ, Amsterdam, The Netherlands

Full list of author information is available at the end of the article
}

[3]. Circulating levels of hsCRP (high sensitive C-Reactive Protein), a biomarker for inflammation, are proven to be a strong, independent predictor of future myocardial infarction and stroke even among apparently healthy asymptomatic men [4]. Finally, in a cohort of healthy individuals, subjects with endotoxin levels beyond $50 \mathrm{pg} / \mathrm{ml}\left(90^{\text {th }}\right.$ percentile) exhibited a threefold increased risk of cardiovascular events (OR [95\% CI], 2.9 [1.4-6.3]; $\mathrm{p}<0.01$ ) [5].

In vivo, monocytes bridge inflammation and atherosclerosis. They express TLR4, the receptor for endotoxin and are involved in all key sequelae of atherosclerosis $[5,6]$. 
We hypothesized that endotoxin exposure in vivo results in changes in monocyte transcriptome that could lead to a more atherogenic phenotype. This would be reflected by differential expression of, among others, atherosclerosis related genes. We aimed to identify these atherosclerosis related genes as candidates for further investigation.

\section{Methods \\ In Vivo Experiments \\ Endotoxin infusion in healthy volunteers}

In order to mimic in vivo systemic inflammation associated with endotoxemia, we used a model in which healthy volunteers are exposed to lipopolysaccharide (LPS), the endotoxin derived from gram negative bacteria [7]. Informed consent was obtained from healthy Caucasian male volunteers for the study, which was approved by the Institutional Review Board of the Academic Medical Center Amsterdam. Inclusion criteria included: no history of sepsis or CVD; not having previously received endotoxin intravenously; non-smokers, no use of any medication and free from any febrile illness in the month preceding the study. In all subjects, a medical history, physical examination, routine laboratory examination, electrocardiogram and chest X-ray were performed. All experiments were performed after an overnight fast.

A bolus of Escherichia coli endotoxin (LPS; catalog number 1235503, lot G2B274; Pharmacopeial Convention, Inc, Rockville, USA; $1 \mathrm{ng} / \mathrm{kg}$ ) was infused intravenously in healthy volunteers. For controls, an equal amount of endotoxin-free $0.9 \% \mathrm{NaCl}$ (saline) was infused. Vital signs, including temperature of the study subjects, were monitored at the Intensive Care Unit by a medical doctor who was present throughout the experiments. The incidence, time and severity of clinical symptoms associated with endotoxemia, were recorded. Blood was regularly sampled for clinical chemistry and hematological parameters. IL-6 levels were determined using the Cytometric Bead Array technique (R\&D systems, Minneapolis, MN, USA). Whole blood samples in $4 \%$ trisodium citrate were obtained at baseline $(\mathrm{T}=0)$, one hour $(\mathrm{T}=1)$ and four hours $(\mathrm{T}=4)$ after LPS infusion.

\section{Monocyte mRNA preparation}

Whole blood was centrifuged 12 minutes at $300 \mathrm{~g}$ and plasma was replaced with an equal volume of PBS buffer containing 1.25\% BSA and 2 mM EDTA (PBS/BSA/ EDTA). The samples were then layered onto 0.5 volume Histopaque-1077 (Sigma-Aldrich, St. Louis, MO, USA) and centrifuged at $400 \mathrm{~g}$ for 20 minutes. Mononuclear cells were removed and washed twice with PBS/BSA/ EDTA buffer. An aliquot of 1 million peripheral mononuclear blood cells were removed for flow cytometric analysis. The remaining cells were used for monocyte isolation using magnetic CD14+ microbeads (Miltenyi Biotech GMBH, Bergisch Gladbach, Germany) according to the manufacturer's instructions. Briefly, $20 \mu \mathrm{l}$ microbeads and $80 \mu \mathrm{l}$ PBS/BSA/EDTA containing $10^{6}$ cells were mixed and incubated on ice for 15 minutes. Cells were washed with PBS/BSA/EDTA before being run on an MS column on a varioMACS (Miltenyi Biotech, Cologne, GMBH). Cell purity was assessed on an aliquot of cells by flow cytometry using anti-CD14 (CLB-mon/1, 8G3, Mouse IgG2a, Sanquin Reagents, Amsterdam, The Netherlands). The purified cells were then lysed in RNA Bee (TelTest Inc., Friendswood, Texas, USA) and RNA extracted according to manufacturer's protocol. The concentration of purified RNA was quantified using a NanoDrop spectrophotometer (NanoDrop Technologies, Wilmington, Delaware, USA) and quality assessed using an Agilent 2100 Bioanalyzer (Agilent, Palo Alto, CA, USA). RNA purity was assessed by Real-Time PCR (RT-PCR) using Taqman transcripts specific for B-cells (CD19/Hs00174333_m1), T-cells (CD3/Hs00167894_m1), erythroid cells (CD235a/ Hs00266777_m1), platelets (CD41/HS00166246_m1), neutrophils (CD66a/Hs00174351_m1), monocytes (CD14/Hs00169122_g1) and GAPDH (Hs99999905_m1) as control. A total of 12 ng of RNA was reverse transcribed and amplified using Taqman Transcription Reagents according to the manufacturer's protocol (Applied Biosystems, Foster City, CA, USA). Assays were run on an Mx4000 Multiplex Quantitative PCR System (Stratagene Inc, La Jolla, California, USA). Only those samples with a FACS purity $>80 \%$ were processed to ensure reliability of results.

\section{Monocyte cDNA preparation}

Template Switching Polymerase Chain Reaction (TSPCR) was used to prepare amplified double stranded (ds) copy DNA (cDNA) following manufacturer's instructions (BD Biosciences Clontech, Palo Alto, CA, USA). Amplifications were performed with either $10 \mathrm{ng}$ (23 cycles) or $100 \mathrm{ng}$ (17 cycles) of starting material, as described previously [8]. Amplified cDNA was subsequently purified using the QIAquick PCR Purification Kit (QIAGEN Inc., Chatsworth, UK) according to manufacturer's protocol with two amendments. Firstly, samples were washed three times in $0.7 \mathrm{~mL}$ PE buffer. Secondly, for elution, $50 \mu \mathrm{L}$ of sterile water was added, the column was left to stand for 2 minutes and then centrifuged. Purified cDNA was quantified using a NanoDrop and fragment length assessed on 1.0\% agarose gel. Performance of the amplification was assessed using RT-PCR as above.

\section{Microarray processing}

Out of the 11 LPS infusion experiments the 5 samples with the highest purity based on FACS and RT-PCR 
and sufficient yield were chosen for arraying at $\mathrm{T}=0 \mathrm{vs}$. $\mathrm{T}=1$ and $\mathrm{T}=0$ vs. $\mathrm{T}=4$. Similarly, 4 out of the 5 were chosen for the control experiments for the $\mathrm{T}=0$ vs. $\mathrm{T}=$ 1 and 2 of the 5 samples for the $\mathrm{T}=0$ vs. $\mathrm{T}=1$. A total of $250 \mathrm{ng}$ of amplified cDNA was labelled by incorporation of Cy3 or Cy5 dCTP using the Bioprime Labelling Kit (Invitrogen Ltd, Paisley, UK). Labelled products were purified on Autoseq G-50 columns (GE Healthcare, Buckinghamshire, United Kingdom) before differential labelling. Biologically paired pre- and post-infusion samples were pooled and hybridized to Hver 2.1.1 cDNA micro-arrays (Wellcome Trust Sanger Institute; WTSI). Hybridization and washing of the arrays were performed according to WTSI protocols. Processed slides were promptly scanned at $10-\mu \mathrm{m}$ resolution on an Agilent Micro-array Scanner (G2505B; Agilent Technologies, Stockport, United Kingdom). Images were exported into GenePix version 4.1 (Molecular Devices, Sunnyvale, CA, USA) for spotfinding and feature extraction. In accordance with MIAME (Minimum Information About a Micro-array Experiments) regulations, all data were deposited into ArrayExpress database at http://www.ebi. ac.uk $[9,10]$.

\section{Data analysis}

Data was analyzed using R-project version 2.2.0. Features were included if all of the following criteria were met: (i) Manually unflagged in Genepix 6.0 (Axon Instruments, Foster City, CA, USA); (ii) ( $\Delta$ medianmean)/median intensity difference $<0.1$;

(iii) Saturation < 20\%; (iv) Mean/standard deviation (SD) > 2; (v) Intensities of background (BG) + 1SD > $90 \%$ and $\mathrm{G}+2 \mathrm{SD}>80 \%$. In addition, the feature had to be either up or down regulated in at least $50 \%$ of the arrays, in order to pass the inclusion threshold. LPS and control samples were analysed separately in a linear mixed effects model with corrections for false discovery rates (FDR) As cut-off we selected a FDR $<0.005$, a posterior probability of differential expression $>0.95$. The foldchanges had to be greater than two and present in at least $70 \%$ of the arrays.

\section{Microarray results validation}

RT-PCR was used to confirm the observations from the comparative microarray study in the 6 LPS samples not used for Microarray for the following transcripts chosen at random: SLA1 (Hs00153504_m1); BATF (Hs00232390_m1); C3aR1 (Hs00377780_m1); AKIP (Hs00610917_g1); ILRN (Hs00174099_m1); TIMP1 (Hs00171558_m1); VCAN (Hs00171642_m1).

RNA (100 ng) was reverse transcribed using the TaqMan RT kit (Applied Biosystems), from which $0.5 \mathrm{ng}$ cDNA was then used as template for RT-PCR following the manufacturer's instructions (Applied Biosystems). Reactions were incubated at $50^{\circ} \mathrm{C}$ for 2 minutes then $95^{\circ}$ $\mathrm{C}$ for 10 minutes, and $\mathrm{RT}-\mathrm{PCR}$ reactions were performed over 40 cycles $\left(95^{\circ} \mathrm{C}\right.$ for 15 seconds, $60^{\circ} \mathrm{C}$ for 1 minute) on an MX-4000 (Stratagene, La Jolla, CA). Threshold values $(\mathrm{Ct})$ were normalized to GAPDH to allow comparison between samples.

\section{In Vivo and In Vitro Data Synthesis}

Datamining in silico of the differentially expressed genes was performed using public databases and tools including biomart, iHOP, pubmed and reactome. Data from the in vitro study was used to select genes which were differentially expressed in both the in vivo and in vitro experiments (see additional file 1, supplementary methods).

\section{Results}

\section{In Vivo Experiments}

\section{Endotoxin infusion in healthy volunteers}

In total, 16 healthy men with an average age of $23 \pm 1$ years (mean $\pm \mathrm{SD}$ ) meeting the inclusion criteria participated; 11 for LPS infusion and five as controls. Medical history, physical examination, routine laboratory examination, electrocardiogram and chest X-ray were normal. All subjects receiving LPS experienced a clinical response consisting of nausea, malaise, chills, muscle ache and fever. Plasma/Serum IL-6 increased significantly (Figure 1). No such effects were observed in the control group.

\section{Monocyte cDNA preparation}

The monocyte count differed significantly between LPS and control experiments at the first hour $(\mathrm{p}<0.05)$ (Figure 2). In the LPS infusion experiments, monocyte count dropped $55 \%$ in the first hour followed by a slow

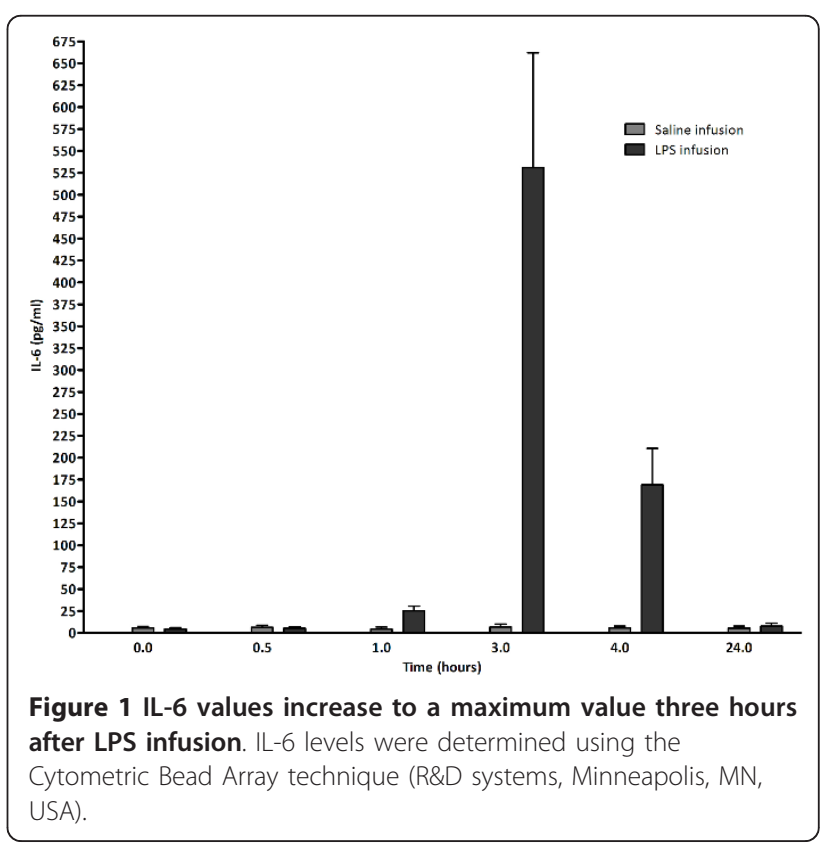




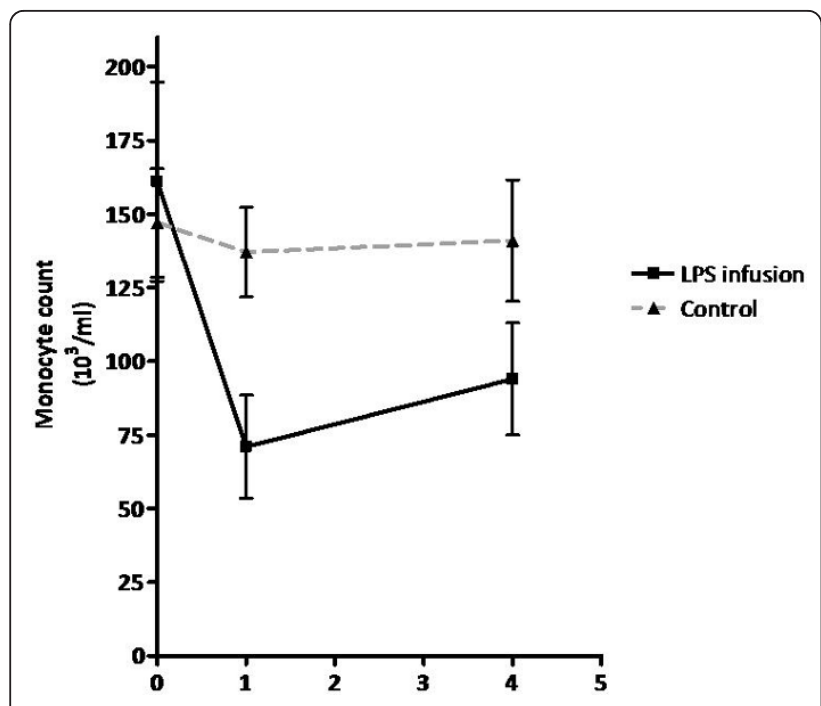

Figure 2 Monocyte count is significantly lower in the LPS infusion experiments compared to saline at $\mathrm{T}=1$.

increase towards normal at four hours. In the control experiments, monocyte count varied slightly but did not decrease as observed after LPS infusion (Figure 2). Summary data for all samples and those included in the study is shown in additional file 2 and 3; supplementary table 1 and 2. RT-PCR performed pre- and post-amplification demonstrated minimal contamination of selected RNA samples by other cell types and non-biased amplification (Data not shown).

\section{Microarray processing}

For the $\mathrm{T}=0$ vs. $\mathrm{T}=1$ experiments, 10 arrays were hybridised with LPS-stimulated samples and 8 with control samples. On average $19 \%$ of spots passed the stringent quality control criteria. One hour after LPS infusion $(\mathrm{T}=1), 11$ genes with a known function were differentially expressed, of which, 1 was down regulated and 10 unregulated (Table 1 and Figure 3) with foldchanges ranging from 2.0 to 5.8 .

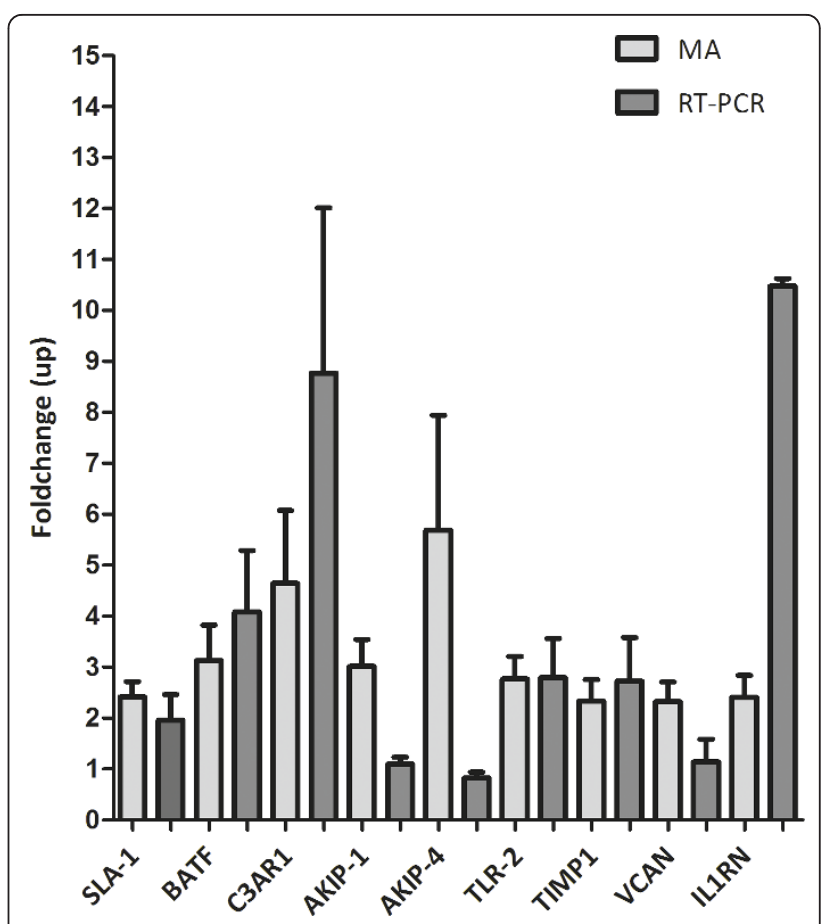

Figure 3 Except for AKIP, microarray (MA) and RT-PCR fold changes are comparable. AKIP-1; Differential expression of AKIP in one hour post infusion samples. AKIP-4; Differential expression of AKIP in four hours post infusion samples.

For the $\mathrm{T}=0$ vs. $\mathrm{T}=4$ experiments, 10 arrays were hybridised with LPS-stimulated samples and 4 arrays with control samples. On average $26 \%$ of spots passed the stringent quality control criteria. In total 28 genes were identified as being differentially expressed in monocytes following LPS stimulation, of which 3 were down regulated and 25 up regulated (see Table 2 and Figure 3). The fold changes ranged from 2.0 to 5.7. Interestingly, $A K I P$ was found to be differentially expressed at both one and four hours after LPS infusion.

Table 1 Genes differentially expressed one hour after LPS infusion

\begin{tabular}{|c|c|c|c|c|}
\hline Ensembl ID & HUGO genelD & Full name & foldchange & p-value \\
\hline ENSG00000172409 & CLP1 & Pre-mRNA cleavage complex II protein Clp1 & 0,47 & 0,001 \\
\hline ENSG00000197766 & CDF & Complement factor D precursor & 2,02 & 0,003 \\
\hline ENSG00000175592 & FOSL1 & Fos-related antigen 1 & 2,05 & 0,001 \\
\hline ENSG00000175602 & DIPA & Delta-interacting protein A & 2,05 & 0,001 \\
\hline ENSG00000166008 & MAGEA9 & Melanoma-associated antigen 9 & 2,37 & 0,001 \\
\hline ENSG00000136689 & IL1RN & Interleukin-1 receptor antagonist protein precursor & 2,41 & 0,003 \\
\hline ENSG00000179271 & PLINP-1 & Papillomavirus L2-interacting nuclear protein 1 & 2,69 & 0,002 \\
\hline ENSG00000181667 & PTPRCAP & Protein tyrosine phosphatase receptor type C-associated protein & 2,85 & 0,005 \\
\hline ENSG00000175756 & AKIP & Aurora kinase A-interacting protein & 3,01 & 0,001 \\
\hline ENSG00000196783 & CCL3L1 & Small inducible cytokine A3-like 1 precursor & 3,25 & 0,002 \\
\hline ENSG00000129277 & CCL4 & Small inducible cytokine A4 precursor & 5,87 & 0,001 \\
\hline
\end{tabular}

No genes were differentially expressed in the controls at $T=0$ vs. $T=1$. 
Table 2 Genes differentially expressed four hours after LPS infusion

\begin{tabular}{|c|c|c|c|c|}
\hline Ensembl ID & HUGO genelD & Full name & foldchange & p-value \\
\hline ENSG00000168383 & HLA-DPB1 & HLA class II histocompatibility antigen, DP(W4) beta chain precursor & 0,44 & $4,2 \mathrm{E}-05$ \\
\hline ENSG00000152518 & ZFP36L2 & Butyrate response factor 2 & 0,47 & $6,7 \mathrm{E}-05$ \\
\hline ENSG00000158050 & DUSP2 & Dual specificity phosphatase 2 & 0,47 & $9,3 \mathrm{E}-04$ \\
\hline ENSG00000148218 & ALAD & Delta-aminolevulinic acid dehydratase & 2,04 & $8,2 \mathrm{E}-04$ \\
\hline ENSG00000152684 & PELO & Pelota homolog & 2,08 & $6,0 \mathrm{E}-04$ \\
\hline ENSG00000163220 & S100A9 & Calgranulin B & 2,09 & $3,8 \mathrm{E}-03$ \\
\hline ENSG00000088986 & DNCL1 & Dynein light chain 1, cytoplasmic & 2,15 & $8,2 \mathrm{E}-04$ \\
\hline ENSG00000134802 & SLC43A3 & Solute carrier family 43 , member 3 & 2,17 & $3,1 \mathrm{E}-03$ \\
\hline ENSG00000163754 & GYG & Glycogenin-1 & 2,21 & $1,1 \mathrm{E}-03$ \\
\hline ENSG00000084733 & RAB10 & Ras-related protein Rab-10 & 2,23 & $9,0 \mathrm{E}-04$ \\
\hline ENSG00000137312 & FLOT1 & Flotillin-1 & 2,23 & $2,6 \mathrm{E}-03$ \\
\hline ENSG00000023330 & ALAS1 & 5-aminolevulinate synthase, nonspecific, mitochondrial precursor & 2,24 & $1,6 \mathrm{E}-03$ \\
\hline ENSG00000015475 & $\mathrm{BID}$ & $\mathrm{BH} 3$ interacting domain death agonist & 2,27 & $2,6 \mathrm{E}-03$ \\
\hline ENSG00000123405 & NFE2 & Transcription factor NF-E2 45 kDa subunit & 2,27 & $2,9 \mathrm{E}-03$ \\
\hline ENSG00000096238 & CLIC1 & Chloride intracellular channel protein 1 & 2,29 & $4,8 \mathrm{E}-04$ \\
\hline ENSG00000168439 & STIP1 & Stress-induced-phosphoprotein 1 & 2,29 & $3,1 \mathrm{E}-03$ \\
\hline ENSG00000101310 & SEC23B & Protein transport protein Sec23B & 2,32 & $6,0 \mathrm{E}-04$ \\
\hline ENSG00000106211 & HSPB1 & Heat-shock protein beta-1 & 2,33 & $2,1 \mathrm{E}-03$ \\
\hline ENSG00000102265 & TIMP1 & Metalloproteinase inhibitor 1 precursor & 2,33 & $3,1 \mathrm{E}-03$ \\
\hline ENSG00000170458 & CD14 & Monocyte differentiation antigen CD14 precursor & 2,40 & $4,8 \mathrm{E}-04$ \\
\hline ENSG00000155926 & SLA & SRC-like-adapter & 2,41 & $1,9 \mathrm{E}-04$ \\
\hline ENSG00000109971 & HSPA8 & Heat shock cognate $70 \mathrm{kDa}$ protein & 2,45 & $4,6 \mathrm{E}-04$ \\
\hline ENSG00000038427 & VCAN & Versican core protein precursor & 2,45 & $8,5 \mathrm{E}-06$ \\
\hline ENSG00000106803 & SC61B & Protein transport protein Sec61 beta subunit & 2,46 & $8,9 \mathrm{E}-05$ \\
\hline ENSG00000137462 & TLR2 & Toll-like receptor 2 precursor & 2,77 & $2,5 \mathrm{E}-04$ \\
\hline ENSG00000156127 & BATF & ATF-like basic leucine zipper transcriptional factor B-ATF & 3,13 & $2,3 \mathrm{E}-04$ \\
\hline ENSG00000171860 & C3aR1 & C3a anaphylatoxin chemotactic receptor & 4,65 & $1,0 \mathrm{E}-03$ \\
\hline ENSG00000175756 & AKIP & Aurora kinase A-interacting protein & 5,68 & 8,7 E-04 \\
\hline
\end{tabular}

No genes were differentially expressed in the controls at $T=0$ vs. $T=4$.

We did not identify any genes that were significantly differentially expressed in the control experiments at either $\mathrm{T}=1$ or $\mathrm{T}=4$ confirming that the differential expression observed, whilst limited, is associated with LPS infusion.

\section{Microarray data validation}

Using quantitative RT-PCR, we were able to confirm the differential expression of 7 of 8 genes tested (Figure 3). Validation was performed on RNA from individuals who had not been part of the subset analysed by microarray, thus highlighting that the changes observed are universal. Absolute fold changes varied between the microarray and Taqman results, however in all cases, except for AKIP (both at $\mathrm{T}=1$ and $\mathrm{T}=4$ ), the direction of differential expression was conserved.

\section{In Vitro Experiments}

In the in vitro experiments a total 1127 genes were differentially expressed due to stimulation with LPS (ArrayExpress accession number E-TABM-483). Six of these genes were also differentially expressed in our in vivo study. For all results see additional file 4, supplementary table 3.

\section{Discussion}

We applied a stepped approach in our study to provide evidence that endotoxin exposure in vivo results in a proatherogenic phenotype in circulating monocytes. First, we proved that the LPS infusion model we applied results in both clinical symptoms and increased IL-6, a marker for inflammation associated with TLR4 signaling (17), with IL-6 levels peaking at three hours post LPS infusion. Second, circulating monocytes were profiled on cDNA arrays pre and post LPS infusion. We generated a list of 39 genes differentially expressed at one or four hours post LPS infusion. A lack of overlap between genes at both time points was observed, with only AKIP being differentially expressed at both time points. This observation is likely due to the sequential activation of pathways after the LPS stimulus. In addition, this might reflect different populations of monocytes contributing to the transcriptome, since only $45 \%$ of monocytes 
remain in circulation one hour after LPS stimulation. Of these genes, 7 of 8 randomly selected candidates were confirmed by RT-PCR. Finally, comparisons with our in vitro data identified 6 overlapping genes $(B A T F, B I D$, C3aR1, IL1RN, SEC61B and SLC43A3). Since C3aR1 has been previously associated with inflammation and atherosclerosis this might be one of the interesting candidates in our experiments to link both disorders. We will focus our discussion on the genes which were differentially expressed in both our in vivo and in vitro experiments.

The C3aR1 gene is located on chromosome 12. The gene product is a highly ligand-specific membrane receptor belonging to the family of the seven transmembrane domain G-protein-coupled receptors. C3aR1 is expressed in monocytes, macrophages and endothelial cells. Binding of $\mathrm{C} 3$ to $\mathrm{C} 3 \mathrm{aR} 1$ induces a wide rang of inflammatory and immune effects [11]. There are multiple lines of evidence for the role of C3aR1 in atherosclerosis.

At gene expression level, in samples of patients with advanced peripheral artery disease (PAD) the gene was expressed at a five times higher level in advanced compared to intermediate atherosclerotic lesions [12]. At protein level, advanced human coronary atherosclerosis plaques express $C 3 a R 1$ in contrast to normal coronary intima [13]. In addition, signaling via $C 3 a R 1$ promotes plaque instability and can thus result in clinical sequelae of acute coronary syndromes [13].

The direct causal role of $C 3 a R 1$ is provided by the fact that pertubaration of this gene in knock out mice models result in significant decrease in atherosclerotic lesion size [14].

Putting the evidence together, we speculate the following sequence of events. We realize that several pathways will co-exist and this is merely one of them. Low grade inflammation due to endotoxinemia results in the higher expression of C3aR1 in vivo. Increased C3aR1 expression in turn results in a proatherogenic monocyte phenotype. Firstly, this results in increased atherosclerotic lesion size. Secondly, it results in increased plaque instability. Ultimately all these effect might result in more clinical CVD events.

The up regulation of $I L 1 R N$ and $B A T F$, in contrast, does not support our hypothesis of the circulating monocyte with a proatherogenic phenotype.

$I L 1 R N$ is a cytokine gene located on chromosome 2 . It is a negative regulator of IL-1 signalling and plays a role as an anti-inflammatory cytokine in acute and chronicinflammation of the vascular wall. It is expressed by macrophages, endothelial cells and smooth muscle cells. Endogenous IL-Ra suppresses atherosclerosis in humans. Decreased expression and not increased expression has been associated with atherosclerotic plaques in mice [15]. The increased expression of IL1RN might be an internal protective mechanism to downlplay the effects of systemic inflammation due to the LPS stimulus.

$B A T F$ is a nuclear basic leucine zipper protein and a member of the AP-1 family of transcription factors located on chromosome 14. It directly regulates key components of the formation and function of follicular helper $\mathrm{T}$ cells and antibody class switching in B cells [16]. In B-cells the expression of BATF is also induced by LPS and IL-6 [16]. Expression of BATF in rat fibroblast suppressed the production of Metalloproteinase (MMP)-2 and MMP-9. MMPs are key players in atherothrombosis due to their extracellular matrix remodelling properties and their functional effects on cells involved in atherogenesis and atherosclerotic complications [17].

The role of BID, SEC61B, SLC43A3 in atherosclerosis is not yet established.

$B I D$ is located on chromosome $22 \mathrm{q}$ and is a critical mediator of inflammation and innate immunity [18]. Mechanistically, BID interacts with NOD1, NOD2 and the $\mathrm{I} \kappa \mathrm{B}$ kinase (IKK) complex, impacting NF- $\kappa \mathrm{B}$ and extracellular signal-regulated kinase (ERK) signalling [18]. Targeting BID by small molecules has been proposed as a way to treat immune-mediated inflammatory disease including inflammatory bowel disease.

$S E C 61 B$ is located on chromosome 9q22. In the Endoplasmatic Recticulum (ER) membrane, the heterotrimeric Sec61 complex comprises three transmembrane subunits (Sec61 $\alpha$, Sec61 $\beta$, and Sec61 $\gamma$ in mammals) and forms protein-conducting channels, collectively termed a translocon. Sec61 $\alpha$ is known to be stabilized by Sec61 $\gamma$ and mainly responsible for the translocation activity in the ER. In contrast to the other two subunits, Sec61 $\beta$ can be stable on its own, and its function is not as well defined. It is known that Sec61 $\beta$ in the inner nucleus membrane (INM) is required for the release of epidermal growth factor from the INM to the nucleus [19].

SLC43A3 is located on chromosome 11 and is known to be highly expressed in macrophages. It is thought to function as a transporter of metabolites and nutrients that are necessary during developmental events, such as organogenesis [20]. It is part of the specific expression pattern of the micro vascular endothelium and has been proposed as putative drug target to pathological angiogenesis [21].

Our study has several limitations. The experiments were conducted in a small number of individuals. However the results are supported by the fact that the selected candidate genes were validated by RT-PCR. In addition, several genes have been previously described in the literature as being differentially expressed in response to endotoxin exposure $[22,23]$. In our experiment we determined the effect of LPS on gene expression in circulating monocytes. However, a substantial part of the monocytes migrated into the vessel wall, 
reflected by a fall in monocyte count in the peripheral blood after LPS infusion. Therefore it begs questioning how representative these circulating monocytes are for the entire population of these cells [24]. In this respect, the overlap in genes differentially expressed in our in vivo study compared to previous in vitro LPS challenges, lends further support to the validity of the identified candidate genes in circulating monocytes. However, the low level of homology between in vivo and in vitro transcriptomes makes us question the value of studying expression profiles of circulating cells as (prognostic) markers for disease states. The poor overlap between in vivo and in vitro experiments could be due to either the intrinsic differences between the two systems or due to the small sample sizes in both studies.

Another limitation is that the procedure of monocyte isolation is time consuming and might thus effect transcription and stability. To minimize these potential effects, for all in vivo and in vitro experiments the same optimized protocol was used by the same analyst. Furthermore genes differentially expressed due to handling would have showed up in the placebo experiments. No genes were differentially expressed in these experiments.

Finally, in comparison to in vitro experiments only a small number of genes were differentially expressed. This is in part due to the stringent quality control criteria we applied during the analysis, the limited coverage of the microarrays and the fact that TLR4 signaling is modulated in vivo by a number of specific pathways.

\section{Conclusion}

Endotoxin exposure in in vivo to healthy individuals identified several candidate genes through which systemic inflammation can result in accelerated atherosclerosis. Out of these candidates, C3aR1, might be a promising target, solely based on the existing literature. Our results in combination with previous reports support the possible role of anaphylatoxins and the complement system as potential target for treatment of acute sequels of atherosclerosis through plaque stabilization.

\section{Additional material}

Additional file 1: Supplementary Methods. Full methods of the in vitro experiments.

Additional file 2: LPS Supplementary Table 1. RNA yield and quality data for all samples and those included in the study.

Additional file 3: LPS Supplementary Table 2. cDNA quantity, quality, labeling and amplification data for all samples and those included in the study.

Additional file 4: Supplementary Table 3 Ctrl vs LPS in vitro. Full results of the in vitro experiments.

\begin{abstract}
Acknowledgements
None

Funding

This project was funded by the Bloodomics consortium, European Union $6^{\text {th }}$ Framework Programme (LSHM-CT-2004-503485) and a Dutch Government ZonMW-VENI Grant 2008 (016.096.044).
\end{abstract}

\section{Author details}

'Department of Vascular Medicine, Academic Medical Center, Meibergdreef 15, 1105 AZ, Amsterdam, The Netherlands. 'Department of Hematology, University of Cambridge, Long Road, CB2 OPT, Cambridge, UK. ${ }^{3}$ Department of Human Genetics, Wellcome Trust Sanger Institute, Hinxton, CB10 1SA, Cambridge, UK. ${ }^{4}$ Department of Experimental Immunohematology, Sanquin Research, Plesmanlaan 125, 1066 CX, Amsterdam, The Netherlands.

${ }^{5}$ Department of Statistics, University of Leeds, Woodhouse lane, LS2 9JT, Leeds, UK. ${ }^{6}$ National Health Service Blood and Transplant, Cambridge, Long Road, CB2 OPT, UK.

\section{Authors' contributions}

WHO, NW and JJZ designed the study. SS, RF, RB and MN designed and carried out the experiments. AF performed the statistical analysis. CL and NW supervised the experiments. SS, NW and MDT wrote the manuscript. SS, RF, AG, NW and SM revised the manuscript. All authors have read and approved the final manuscript.

\section{Conflict of interest}

The authors declare that they have no competing interests.

Received: 8 October 2010 Accepted: 10 August 2011

Published: 10 August 2011

\section{References}

1. Frostegard J: Systemic lupus erythematosus and cardiovascular disease. Lupus 2008, 17:364-367.

2. Turesson C, Jarenros A, Jacobsson L: Increased incidence of cardiovascular disease in patients with rheumatoid arthritis: results from a community based study. Ann Rheum Dis 2004, 63:952-955.

3. Thompson PA, Tobias PS, Viriyakosol S, Kirkland TN, Kitchens RL: Lipopolysaccharide (LPS)-binding protein inhibits responses to cellbound LPS. J Biol Chem 2003, 278:28367-28371.

4. Ridker PM, Cushman M, Stampfer MJ, Tracy RP, Hennekens $\mathrm{CH}$ : Inflammation, aspirin, and the risk of cardiovascular disease in apparently healthy men. N Engl J Med 1997, 336:973-979.

5. Michelsen KS, Doherty TM, Shah PK, Arditi M: Role of Toll-like receptors in atherosclerosis. Circ Res 2004, 95:e96-e97.

6. Libby P: Inflammation in atherosclerosis. Nature 2002, 420:868-874.

7. van Deventer SJ, Buller HR, ten Cate JW, Aarden LA, Hack CE, Sturk A: Experimental endotoxemia in humans: analysis of cytokine release and coagulation, fibrinolytic, and complement pathways. Blood 1990, 76:2520-2526.

8. Macaulay IC, Tijssen MR, Thijssen-Timmer DC, Gusnanto A, Steward M, Burns P, Langford CF, Ellis PD, Dudbridge F, Zwaginga JJ, et al: Comparative gene expression profiling of in vitro differentiated megakaryocytes and erythroblasts identifies novel activatory and inhibitory platelet membrane proteins. Blood 2007, 109:3260-3269.

9. Brazma A, Hingamp P, Quackenbush J, Sherlock G, Spellman P, Stoeckert C, Aach J, Ansorge W, Ball CA, Causton HC, et al: Minimum information about a microarray experiment (MIAME)-toward standards for microarray data. Nat Genet 2001, 29:365-371.

10. Parkinson H, Kapushesky M, Shojatalab M, Abeygunawardena N, Coulson R, Farne A, Holloway E, Kolesnykov N, Lilja P, Lukk M, et al: ArrayExpress-a public database of microarray experiments and gene expression profiles. Nucleic Acids Res 2007, 35:D747-D750.

11. Jacob A, Bao L, Brorson J, Quigg RJ, Alexander JJ: C3aR inhibition reduces neurodegeneration in experimental lupus. Lupus 2010, 19:73-82.

12. Fu S, Zhao H, Shi J, Abzhanov A, Crawford K, Ohno-Machado L, Zhou J, Du Y, Kuo WP, Zhang J, et al: Peripheral arterial occlusive disease: global gene expression analyses suggest a major role for immune and inflammatory responses. BMC Genomics 2008, 9:369. 
13. Oksjoki R, Laine P, Helske S, Vehmaan-Kreula P, Mayranpaa MI, Gasque P, Kovanen PT, Pentikainen MO: Receptors for the anaphylatoxins C3a and $\mathrm{C} 5 \mathrm{a}$ are expressed in human atherosclerotic coronary plaques. Atherosclerosis 2007, 195:90-99.

14. Yang $X$, Peterson $L$, Thieringer $R$, Deignan JL, Wang X, Zhu J, Wang $S$, Zhong $H$, Stepaniants $S$, Beaulaurier J, et al: Identification and validation of genes affecting aortic lesions in mice. J Clin Invest 2010, 120:2414-2422.

15. Isoda K, Ohsuzu F: The effect of interleukin-1 receptor antagonist on arteries and cholesterol metabolism. J Atheroscler Thromb 2006, 13:21-30

16. Ellyard Jl, Vinuesa CG: A BATF-ling connection between B cells and follicular helper T cells. Nat Immunol 2011, 12:519-520.

17. Busti C, Falcinelli E, Momi S, Gresele P: Matrix metalloproteinases and peripheral arterial disease. Intern Emerg Med 2009.

18. Yeretssian G, Correa RG, Doiron K, Fitzgerald P, Dillon CP, Green DR, Reed JC, Saleh M: Non-apoptotic role of BID in inflammation and innate immunity. Nature 2011.

19. Wang YN, Yamaguchi H, Huo L, Du Y, Lee HJ, Lee HH, Wang H, Hsu JM, Hung MC: The translocon Sec61beta localized in the inner nuclear membrane transports membrane-embedded EGF receptor to the nucleus. J Biol Chem 2010, 285:38720-38729.

20. Stuart RO, Pavlova A, Beier D, Li Z, Krijanovski Y, Nigam SK: EEG1, a putative transporter expressed during epithelial organogenesis: comparison with embryonic transporter expression during nephrogenesis. Am J Physiol Renal Physiol 2001, 281:F1148-F1156.

21. Wallgard E, Larsson E, He L, Hellstrom M, Armulik A, Nisancioglu MH, Genove G, Lindahl P, Betsholtz C: Identification of a core set of 58 gene transcripts with broad and specific expression in the microvasculature. Arterioscler Thromb Vasc Biol 2008, 28:1469-1476.

22. Vandal K, Rouleau P, Boivin A, Ryckman C, Talbot M, Tessier PA: Blockade of S100A8 and S100A9 suppresses neutrophil migration in response to lipopolysaccharide. J Immunol 2003, 171:2602-2609.

23. Marsik C, Mayr F, Cardona F, Derhaschnig U, Wagner OF, Jilma B: Endotoxaemia modulates Toll-like receptors on leucocytes in humans. $\mathrm{Br}$ J Haematol 2003, 121:653-656.

24. Hojman H, Lounsbury D, Harris H, Horn JK: Immunodepressive effects of LPS on monocyte CD14 in vivo. J Surg Res 1997, 69:7-10.

\section{Pre-publication history}

The pre-publication history for this paper can be accessed here: http://www.biomedcentral.com/1755-8794/4/64/prepub

doi:10.1186/1755-8794-4-64

Cite this article as: Sivapalaratnam et al.: Identification of candidate genes linking systemic inflammation to atherosclerosis; results of a human in vivo LPS infusion study. BMC Medical Genomics 2011 4:64.

\section{Submit your next manuscript to BioMed Central and take full advantage of:}

- Convenient online submission

- Thorough peer review

- No space constraints or color figure charges

- Immediate publication on acceptance

- Inclusion in PubMed, CAS, Scopus and Google Scholar

- Research which is freely available for redistribution

Submit your manuscript at www.biomedcentral.com/submit 